Notre Dame Journal of Formal Logic

Volume 43, Number 2, 2002

\title{
The Complexity of Revision, Revised
}

\author{
G. Aldo Antonelli
}

\begin{abstract}
The purpose of this note is to acknowledge a gap in a previous paper, "The complexity of revision," and to provide a corrected version of the argument.
\end{abstract}

The main point of the argument given in Antonelli [1] is to show that the GuptaBelnap revision-theoretic system $\mathbf{S}^{*}$ admits of an analogue of the LöwenheimSkolem theorem for first-order logic (all notation and terminology are defined in Gupta and Belnap [2]). In particular, a crucial step in the argument requires that we show the following.

Proposition 1 If $\varphi$ is false in a model $\mathfrak{M}+h$, where $h$ is a hypothesis reflexive in a revision sequence $\&$ based on $\mathfrak{M}$ (and relative to a jump operation $\delta$ ), then $\varphi$ is false in a countable model $\mathrm{M}^{\prime}+k$, where $k$ is a hypothesis reflexive in a countable revision sequence $\delta^{\prime}$ based on $\mathfrak{M}^{\prime}$ (relative to $\delta$ ).

If this is carried out, then a theorem of McGee ([3], p. 176) guarantees that $k$ is already reflexive at some countable state $\alpha$, that is, $\delta_{\alpha}^{\prime}=k$ for some countable $\alpha>0$, and the argument in [1] shows how to write this as a $\Pi_{2}^{1}$-sentence.

Actually, the argument in [1] purports to show more than Proposition 1, namely, that given a revision sequence $\delta$ based on a model $\mathfrak{M}$, then there is a countable revision sequence $\delta^{\prime}$ based on a countable model $\mathfrak{M}^{\prime}$ such that $\mathfrak{M}+\delta_{\alpha}$ is an elementary extension of $\mathfrak{M I}^{\prime}+\wp_{\alpha}^{\prime}$ for every ordinal $\alpha$ less than the length of $\xi^{\prime}$. Taking $h=\varsigma_{0}$ and $k=s_{0}^{\prime}$ we obtain the claim we need.

The strategy is to begin with a countable elementary submodel $\mathfrak{M}^{\prime}+h^{\prime}$ of $\mathfrak{M}+h$, which exists by the ordinal Löwenheim-Skolem theorem, and for each ordinal $\alpha>0$, find a hypothesis $\delta_{\alpha}^{\prime}$ such that $\mathfrak{M I}^{\prime}+\delta_{\alpha}^{\prime}$ is still elementarily equivalent to (indeed, an elementary submodel of) $\mathfrak{M}+\delta_{\alpha}$. This gives rise to a revision sequence $\delta^{\prime}$ (based on

Received 05/28/98; accepted 03/18/03; printed December 8, 2003

2001 Mathematics Subject Classification: Primary, 03B60

Keywords: revision theory, truth, Löwenheim-Skolem theorem

(c) 2003 University of Notre Dame 
the countable model $\mathfrak{M}^{\prime}$ ) in which $h^{\prime}$ is reflexive (since each $\mathfrak{M}+\delta_{\alpha}$ is an elementary extension of $\left.\mathfrak{M}^{\prime}+\zeta_{\alpha}^{\prime}\right)$.

Naturally, one would like to construct the new revision sequence $\delta_{\alpha}^{\prime}$ by induction on $\alpha$, the case for $\alpha=0$ being served on a silver platter by the Löwenheim-Skolem theorem. The inductive case appears to go through: one shows by induction on $\varphi\left(x_{1}, \ldots, x_{n}\right)$ that $d_{1}, \ldots, d_{n} \in \mathfrak{M}^{\prime}$ satisfy $\varphi$ in $\mathfrak{M}^{\prime}$ if and only if they satisfy the formula obtained from $\varphi$ by replacing each occurrence of the definiendum $G$ by the definiens $A(G)$; and similarly that $d_{1}, \ldots, d_{n} \in \mathfrak{M}^{\prime}$ satisfy $\varphi$ in $\mathfrak{M}$ if and only if they satisfy the formula obtained from $\varphi$ by replacing each occurrence of the definiendum $G$ by the definiens $A(G)$. The elementary equivalence of $M+\delta_{\alpha+1}$ and $\mathfrak{M}^{\prime}+\delta_{\alpha+1}^{\prime}$ then follows from the elementary equivalence of $\mathfrak{M}+\S_{\alpha}$ and $\mathfrak{M}^{\prime}+\delta_{\alpha}^{\prime}$. (The argument in [1] is a little too swift on this point too).

However, the construction breaks down at the limit stage. For $\lambda$ a limit ordinal, we need to find a hypothesis $k=\wp_{\lambda}^{\prime}$ such that $k$ coheres with $\AA_{\beta}^{\prime}$ (for $\beta<\lambda$ ), and $\mathfrak{M}^{\prime}+k$ is an elementary submodel of $\mathfrak{M}+\delta_{\lambda}$. The condition that $\mathfrak{M N}^{\prime}+k$ be an elementary submodel of $\mathfrak{M}+\delta_{\lambda}$ forces us to put $k=\delta_{\lambda} \cap \mathfrak{M}^{\prime}$ and then we are left with the task of showing that the submodel is elementary. Contrary to what is asserted in [1], this cannot be ascertained by induction on $\varphi$, the crucial case being — not surprisinglythe quantifier case. Observe that the two models are known to be equivalent over the base langauge $\mathscr{L}$ and that we want to show that they are still equivalent when the language is expanded with the new predicate symbol $G$. But the only thing we know about the extensions $\delta_{\lambda}$ and $\delta_{\lambda}^{\prime}$ of the predicate $G$ is that they both cohere with the previous stages, and this does not seem enough to push the induction through.

It seems, therefore, that a different approach is called for. But before we set out to explain what that approach might look like, let us observe that the problem would be quickly disposed of if we had a positive answer to the following question.

Question 2 Suppose $\mathfrak{M C}^{\prime}$ is an elementary submodel of $\mathfrak{M}$ (over a language $\mathcal{L}$ ) and suppose $\mathfrak{L}^{+}$is the expansion obtained by adding a 1-place predicate symbol G. Let $A \subseteq \mathfrak{M}$ and suppose $\mathfrak{M}+A$ is the expansion of $\mathfrak{M}$ interpreting $G$ over $A$. Is there a set $B \subseteq \mathfrak{M}^{\prime}$ such that $\mathfrak{M}^{\prime}+B$ is an elementary submodel of $\mathfrak{M}+A$ ?

The following counterexample, due to McGee, shows that the problem has a negative answer: let $\mathfrak{M}$ be a countable nonstandard model of arithmetic and $\mathfrak{M}^{\prime}$ be its standard part. Put $A=\{x \in \mathfrak{M} \mid x$ is nonstandard $\}$, and let $\mathfrak{M}+A$ be the expansion of $\mathfrak{M}$ interpreting $G$ as $A$. Then there cannot be any subset $B$ of $\mathfrak{M i}^{\prime}$ such that $\mathfrak{M}+A$ is an elementary extension of $\mathfrak{M}^{\prime}+B$, for $\mathfrak{M}+A$ verifies all sentences

$$
\begin{aligned}
& \exists x G(x), \\
& \forall x(G(x) \rightarrow 0<x), \\
& \forall x(G(x) \rightarrow s(0)<x), \\
& \ldots \\
& \forall x\left(G(x) \rightarrow s^{n}(0)<x\right), \\
& \cdots
\end{aligned}
$$

We now turn to the problem of finding a solution to the original problem in Proposition 1. As it turns out, the main idea here is to apply the Löwenheim-Skolem theorem "wholesale" to the entire revision sequence and not pointwise only to the first stage of the sequence. 
Here are the details. At the outset, we are given a revision sequence $\&$ based on a model $\mathfrak{M}$ and a hypothesis $h$ that is reflexive in $\delta$; we also assume that a given sentence $\varphi$ is false in $\mathfrak{M}+h$. The already-mentioned result of McGee ([3], p. 176) ensures that under these circumstances $h$ already reappears as $\delta_{\alpha}$ where $\alpha$ is bounded by the cardinality of $\mathfrak{M}$ (or $\aleph_{0}$, if $\mathfrak{M}$ is finite). Therefore, we can assume that $\delta$ itself has length bounded by the cardinality of $\mathfrak{M}$.

What we want now is the following.

Problem 3 Given an ordinal $\alpha$ and a revision sequence $\delta$ of length $\alpha$ based on a model $\mathfrak{M}$ such that the sentence $\varphi$ fails on some hypothesis reflexive in $\delta$, find a revision sequence $\delta^{\prime}$ of countable length $\alpha_{0}$, based on a countable model $\mathfrak{M}^{\prime}$ and a hypothesis $k$ reflexive in $\mathcal{S}^{\prime}$, such that $\varphi$ fails in $\mathfrak{M}^{\prime}+k$.

We consider the sequence of models $\mathfrak{M}+\S_{\beta}$, for $\beta<\alpha$ and find a standard model (S) of set theory in which such a sequence exists as a set. By Löwenheim-Skolem theorem, $(S)$ has a countable elementary submodel $\mathbb{S}^{\prime}$. Then by elementary equivalence the following is true in $\left({ }^{\prime \prime}\right.$ ' since it is true in $(5)$.

There exists a function $f$, a set (i.e., a "model") $M^{\prime}$ and an ordinal $\alpha_{0}$ such that

1. the domain of $f$ is $\alpha_{0}$; and

2. for every $\beta<\alpha_{0}$ the value $f(\beta)$ is a subset of $M^{\prime}$; and

3. each model $M+f(\beta+1)$ is the "jump" of $M^{\prime}+f(\beta)$; and

4. for each limit $\lambda<\alpha_{0}, f(\lambda)$ coheres with all the previous $f(\beta)$; and

5. there is $\beta<\alpha_{0}$ such that $\beta>0$ and $f(\beta)=f(0)$ (i.e., $f(0)$ is reflexive); and

6. $M+f(0) \models \neg \varphi$.

We can define a model $\mathfrak{M}^{\prime}$ having $M^{\prime}$ as universe and a sequence of subsets $\delta^{\prime}$. In order to establish (3) we need to verify that $\delta^{\prime}$ is indeed a revision sequence. Now the coherence condition, as we mentioned, is elementary and therefore preserved. In addition, we need to verify that the ordinals of $\left(S^{\prime}\right.$ are standard.

But the ordinals of $\left(S^{\prime}\right.$ must be standard: for if the ordinals of $(5)$ are standard to begin with, they will still be standard in any submodel of $\left(\mathbb{S}\right.$ and therefore also in $\mathbb{S}^{\prime}$. This ensures that $f^{\prime}$ is a revision sequence "in the real world" and not only in $\left(S^{\prime}\right.$ '. Moreover, since $\left(S^{\prime}\right.$ ' is countable, the ordinal $\alpha_{0}$ giving the length of the new revision sequence will also be countable (even if it is uncountable "from the point of view" of $\left(S^{\prime}\right)$. This establishes (3).

One more point requires attention: as stated, the proof is not formalizable in ZFC because it presupposes the existence of a standard model of ZFC containing the given revision sequence. This is only a worry if we were interested in a ZFC-proof, and not just in a proof. But even this residual worry can be assuaged by requiring the model (S) to be a model of a finite fragment of ZFC that is still large enough to prove the required facts about the revision sequence $\&$.

\section{References}

[1] Antonelli, G. A., "The complexity of revision," Notre Dame Journal of Formal Logic, vol. 35 (1994), pp. 67-72. Zbl 0801.03021. MR 95d:03042. 75, 76 
[2] Gupta, A., and N. Belnap, The Revision Theory of Truth, The MIT Press, Cambridge, 1993. Zbl 0858.03010. MR 95f:03003. 75

[3] McGee, V., Truth, Vagueness, and Paradox, Hackett Publishing Co., Indianapolis, 1990. Zbl 0734.03001. MR 92k:03004. 75, 77

[4] Orilia, F., "Property theory and the revision theory of definitions," The Journal of Symbolic Logic, vol. 65 (2000), pp. 212-46. Zbl 0960.03003. MR 2001i:03057. 78

\section{Acknowledgments}

This gap was originally pointed out by Francesco Orilia (personal communication and Orilia [4]), and the fix was developed in correspondence with Vann McGee.

Department of Logic and Philosophy of Science

3151 Social Science Plaza

University of California, Irvine

Irvine CA 92697-5100

aldo@uci.edu 